\title{
Efectos de la violencia de pareja en mujeres migrantes*
}

\author{
Effects of partner violence in migrant women \\ Recibido: 11 de julio de 2012 - Revisado: 17 de septiembre de 2012 - Aceptado: 30 de enero de 2013
}

\author{
Leonor Guadalupe Delgadillo Guzmán ${ }^{1}$ \\ Agustín Vargas Cortez ${ }^{2}$ \\ Ángela Nievar ${ }^{3}$ \\ José Francisco Argüello Zepeda ${ }^{4}$ \\ Leonor González Villanueva ${ }^{5}$
}

\begin{abstract}
Resumen
Este estudio analiza la relación entre violencia de pareja, depresión y autoeficacia materna en mujeres migrantes. Se considera que este tipo de violencia produce hundimiento anímico y dificulta el desenvolvimiento como madre. Es un estudio cuantitativo de tipo correlacional explicativo, trabajado con 40 mujeres inmigrantes con dos a 25 años como residentes, violentadas con al menos un episodio referido y por lo menos un hijo en preescolar. Se utilizaron tres instrumentos, "The Parental Involvement and Efficacy", "The Survey-Depression Scale" y "The Revised Conflict Tactic Scale”. Los resultados arrojaron importantes relaciones entre las variables, mientras más violencia vivan las mujeres, más depresión, y mientras más depresión, menor autoeficacia materna.
\end{abstract}

\section{Palabras clave}

Violencia de pareja, inmigración femenina, eficacia parental, depresión.

\begin{abstract}
This study analyzes the relationship between partner violence, depression and maternal self-efficacy in migrant women. It is thought that this type of violence brings about sinking morale and a hindrance for their development as mothers. It is a quantitative study of an explanatory correlational type, worked with 40 immigrant women with a residence of two to 25 years, with at least one reported episode of violation and at least one son in preschool. Three instruments were employed: "The Parental Involvement and Efficacy", "The Survey-Depression Scale" and "The Revised Conflict Tactic Scale". The results showed important relationships between the variables, namely, the more violence women suffer, the greater the depression is, and the greater the depression is, the lesser the maternal self-efficacy becomes.
\end{abstract}

Key Words

Partner violence, female immigration, parental effectiveness, depression.

\footnotetext{
" Este estudio es resultado del proyecto de investigación 'Los efectos de la aculturación de la violencia doméstica: un estudio comparativo entre mujeres inmigrantes hispánicas en México y los Estados Unidos', financiado por la Universidad Autónoma del Estado de México. Aquí se reportan los hallazgos cuantitativos en mujeres migrantes en México.

${ }^{1}$ Doctora en Ciencias Sociales, profesora e investigadora de la Facultad de Ciencias de la Conducta de la Universidad Autónoma del Estado de México.

Correo electrónico: delgadilloleonor@gmail.com

${ }^{2}$ Investigador del Colegio de bachilleres del Estado de México.

Correo electrónico: zaicolandia_1689@hotmail.com

${ }^{3}$ Ph.D. Family and Child Ecology, profesora asociada al Departamento de Psicología Educacional de la Universidad del Norte de Texas.

Correo electrónico:

angela.nievar@unt.edu

${ }^{4}$ Doctor en Antropología, profesor asociado de la Facultad de Ciencias de la Conducta de la Universidad Autónoma del Estado de México.

Correo electrónico: farguello2010@hotmail.com

${ }^{5}$ Profesora asociada de la Facultad de Ciencias de la Conducta de la Universidad Autónoma de México.

Correo electrónico:

gveleonor@gmail.com
} 


\section{Introducción}

Este trabajo forma parte de una investigación más amplia titulada 'Los efectos de la aculturación sobre la violencia doméstica: un estudio comparativo entre mujeres hispanas inmigrantes en México y en Estados Unidos', que hace una mayor reflexión sobre esta problemática, desarrolla mayores conocimientos efectivos que ayuden en la configuración de comportamiento para anticipar o desactivar la violencia así como incentivar la equidad. Estudio de corte cuantitativo en el que se utilizaron escalas psicométricas para explorar la violencia de pareja, la depresión y la eficacia materna en mujeres inmigrantes, lo que remite a un sector poblacional particular. Las hipótesis planteadas fueron: la depresión es un elemento psicológico presente en mujeres inmigrantes víctimas de violencia de pareja que viven en el Estado de México; la violencia de pareja y la depresión son factores que influyen en la ineficacia materna en mujeres inmigrantes que viven en el Estado de México.

Boneva \& Frieze (2001) refieren que la inmigración es un fenómeno mundial caracterizado por la búsqueda de mejores oportunidades económicas, implicando importantes adaptaciones en los esquemas de pensamientos, código de valores, motivaciones, prácticas culturales y tomas de decisión, que no solo involucran al individuo sino también a su familia. Estos elementos se ven agudizados por la condiciones de penetración con las que se introduce el sujeto a un nuevo escenario social, si el migrante cuenta con un empleo previamente acordado a su desplazamiento, si tiene un perfil laboral competitivo, si es soltero(a), si es padre o madre de familia, si migra solo o acompañado. La tensión que produce el cambio de residencia se incrementa cuando se trata de una cultura receptora con la que no se comparte no solo la nacionalidad sino también algo tan básico como el idioma.

Trátese de un tránsito local, nacional o internacional, las presiones sociales y psico- lógicas comunes se ven exacerbadas, el estrés aumenta y, en no pocos casos, el estilo de afrontamiento se torna violento, sobre todo contra quienes están más cerca afectivamente, es decir, la pareja y los hijos(as). De esta manera, en algunos casos, la interacción progresivamente se va tornando hostil hasta llegar, eventualmente, a ser violenta: actos dañinos conscientes y voluntarios, ya sean físicos, psicológicos (desvalorización, control, coerción e indiferencia), económicos o sexuales, directos, indirectos o disfrazados, que buscan obtener de la víctima algún tipo de satisfacción (Delgadillo y Mercado, 2010). Incluso en casos extremos la violencia puede transformarse en el estilo de vida del agresor y la víctima(s).

La disposición psicoadaptativa y las habilidades sociales se someten a una fuerte carga para resolver cuestiones elementales, como la casa, la comida, los servicios domésticos, hasta complicadas como el trabajo, la escuela, las redes de apoyo, tiempos y movimientos familiares, entre otros. La falta de resultados favorables junto con la incertidumbre, incrementan el desasosiego, aumentan la ansiedad, estimulan trastornos del sueño, de la alimentación, psicosomáticos, disminuyen la tolerancia, afectan las relaciones sociales, se alteran procesos del pensamiento junto con la aparición de sentimientos adversos como tristeza, abatimiento y desesperación. La violencia, en este sentido, se traduce en un mecanismo negativo para metabolizar esta mezcla dañina para el individuo, en este caso para los inmigrantes, y sus relaciones cercanas (Delgadillo, 2011).

A los receptores de ella se les afecta su estado anímico, particularmente asoma la depresión caracterizada por una profunda tristeza mayor a los dos meses, que deteriora la calidad de vida, el sujeto vive en el sufrimiento, desarrolla una incapacidad para realizar sus actividades personales y sociales (Beck, Rush, Shaw y Emery, 1983; Ferreira, 1989). Se puede advertir que la unión de un movimiento migratorio junto con la violencia 
familiar configuran una negativa fusión en la que las mujeres constituyen el blanco ideal de sus parejas. Taylor, Guterman, Lee \& Rathouz (2009) señalaron que las madres inmersas en relaciones de pareja violentas viven con estrés en el cuidado de sus hijos. Estos últimos igualmente se ven perturbados en su salud psíquica como víctimas indirectas (Campbell, Sullivan \& Davidson, 1995).

Las familias migrantes representan un grupo en el que se comparten generalmente vínculos consanguíneos, afectivos positivos intensos, con un proyecto de vida común, lealtades compartidas e historias personales que decantan lados ordinarios y conocidos por sus miembros. Puede decirse que comparte muchas de las características de las familias no inmigrantes, una dimensión biológica de consanguinidad que puede o no estar presente entre sus miembros; una dimensión psicológica por la que se anidan afectos, experiencias comunes, rutas de vida y proyectos individuales y compartidos; una dimensión sociológica, al constituir un grupo que se inserta en una malla social más grande que ella misma, que la contiene y la reconoce en varias de sus actividades como el trabajo, la educación, la salud y el esparcimiento, entre otros. En pocas palabras, cada uno de sus miembros influencia al resto, conformando un sistema dinámico que tiene la potencia de favorecer $u$ obstaculizar su desarrollo (Satir, 1994).

Se puede decir que la familia, la pareja y los hijos, en general, reproducen, en mayor o menor medida, los roles culturalmente establecidos. Se espera, entonces, que el padre sea capaz de cubrir las necesidades de todos y cada uno, fundamentalmente las materiales; las afectivas correrán a cargo de la madre, y el apego a las prescripciones y proscripciones señaladas por los padres quedan a cargo de los hijos(as). Esta caracterización por género pone en una clara vertical a cada género, por ejemplo la superioridad física del hombre hacia la mujer, que a la postre favorece la producción de la violencia en la pareja (Gilmore, 1995).
El Instituto Nacional de Salud Pública en México (2003) reporta que entre el 10\% y el $73 \%$ de las mujeres manifestaron haber vivido violencia doméstica. Por su parte, el Instituto Nacional de Estadística, Geografía e Informática (Inegi, 2004) reveló que en este mismo país una de cada tres mujeres sufre maltrato de algún tipo por su pareja. Entre sus efectos destaca la depresión, caracterizada por distorsión de la realidad, desinterés por las actividades cotidianas, fatiga, sensación de lentitud, problemas de concentración, trastornos del sueño, sentimientos de culpa, inutilidad, desesperanza, aumento o pérdida de peso, disminución del deseo sexual, ideación suicida y pensamientos negativos sobre sí mismo(a), entre otros (Beck, et al., 1983).

El estado depresivo puede durar de forma ininterrumpida semanas o meses, el cuadro de gravedad depresiva es vivido por el afectado con sufrimiento y le impide realizar sus actividades personales y sociales, en donde la afectada apenas puede reaccionar o no a una dedicación especial de sus familiares. Taylor, Guterman, Lee \& Rathouz (2009) afirman que las madres inmersas en relaciones maritales violentas sufren más estrés en el cuidado de sus hijos(as), por otro lado, niños(as) que han observado el uso de la violencia como una forma de resolver conflictos experimentan resultados negativos (Campbell, et al., 1995).

La violencia de género afecta a la sociedad ya que, en sus múltiples formas, la violencia pone en peligro la salud de todos los involucrados, disminuye las defensas y la inmunidad orgánica, reduce o hace más rígidas las defensas psíquicas, incrementa las enfermedades existentes y produce nuevas alteraciones, limita las capacidades de participación en la familia. La violencia de género causa más muertes e incapacidad entre las mujeres de 15 a 44 años que el cáncer, la malaria, los accidentes de tráfico e incluso la guerra (Álvarez, 2003).

La comunicación se ve deteriorada, los esquemas de diálogo se producen a través de 
comentarios incompletos, distorsionados o generalizados con una carga sutil o explícita de hostilidad (Satir, 1994). El desempeño materno-paterno es hasta cierto punto distante, pasivo en el involucramiento con las actividades de los hijos(as), dedicándose fundamentalmente a controlar el cumplimiento exacto de los mensajes transmitidos cargados de principios, valores, tradiciones y consignas prescriptivas incuestionables (Minuchin, 2004).

Con este patrón de ejecución parental las relaciones se mantienen asimétricas, no se rompe la jerarquía, se mantiene una distancia emocional que dificulta el acercamiento afectivo entre padres-madres e hijos(as), incluso entre los mismos hermanos porque replican el modelo de sus propios padres que lleva implícita la marca de la desigualdad según el sexo. Este modelo de relación impide proyectar las potencialidades de cada uno de los miembros, porque se está ocupado(a) en vigilar que las actividades familiares corran como deben ser. De esta manera se inhiben las posibilidades de enriquecerse mutuamente ante la presencia vivificante de los demás.

Una parte importante de este tipo de comportamientos inadecuados obedece a la influencia de la cultura con el peso histórico que le acompaña, traduciéndose en un denso legado, que a primera vista se antoja inamovible. Ubicando en las estructuras psíquicas de los individuos preceptos aparentemente justificables, esta es la cruz que te tocó cargar; es que tu eres mujer y él es hombre, entre otros. Un conjunto de ideaciones que favorecen la producciónreproducción de la violencia de género de la que no escapan las parejas que dan origen a sus familias.

El estudio realizado por Heise y GarcíaMoreno (2003) apunta que el 69\% de las mujeres entrevistadas refirieron haber sido agredidas físicamente por sus parejas en algún momento de sus relaciones. Los datos de varios países indican que esta violencia es la causa de un nú- mero significativo de decesos entre las mujeres, porque terminan siendo asesinadas por sus parejas. En Australia, Canadá, Estados Unidos y Sudáfrica, hasta $70 \%$ de los asesinatos de mujeres fueron cometidos por sus esposos o novios, a menudo en el marco de una relación de maltrato constante. Jacobson y Gottman (2001) señalan que en Estados Unidos, cada año, al menos 1,6 millones de mujeres casadas son objeto de graves agresiones de sus esposos y que al menos una tercera parte de todas las mujeres asesinadas han muerto a manos de sus maridos, exmaridos, novios o exnovios.

En este sentido, los niños que crecen en hogares violentos aprenden e interiorizan una serie de creencias y valores negativos sobre las relaciones con otros y, especialmente, sobre las relaciones familiares y sobre la legitimidad del uso de la violencia como método válido para la resolución de conflictos, fruto todo ello de la interacción tanto de factores culturales y sociales (socialización diferencial de género y aceptación social del uso de la violencia) como situacionales (historia de violencia intrafamiliar) (Patró, Limiñana y Martínez, 2003). La violencia, en síntesis, representa una forma de conducta de género arraigada en la conducta aprendida y aceptada por grandes segmentos de la población, en tanto que existe una valoración discriminada de la masculinidad y la feminidad.

Los efectos sobre la(s) víctima(s) son inquietantes, tristeza vital y lentitud psicomotriz definen con exactitud lo esencial de la depresión. No se podría establecer el diagnóstico si no se dan estos dos elementos. Hay otros síntomas que se dan con frecuencia, pero sin el mismo carácter de constancia y sin la misma coherencia lógica de los precedentes. Se trata de los trastornos del sueño (insomnio en general, sobre todo hacia el final de la noche, pero otras veces refugio en el sueño), de las anomalías del comportamiento alimenticio (anorexia acompañada de adelgazamiento, pero a veces bulimia con aumento de peso). Trastornos neurovegetativos y perturbaciones funcionales de los órga- 
nos pueden acompañar o preceder la evolución del síndrome depresivo. Así como manifestaciones físicas de la angustia (constricción cervical o torácica, vértigos), trastornos del ritmo cardiaco, baja de la tensión arterial, trastornos digestivos y dolores (Widlöcher, 1986).

Solo se habla de una enfermedad depresiva cuando el trastorno es tan intenso o de tan larga duración que se convierte en una grave carga personal o social. Los siguientes puntos de referencia indican una enfermedad: el estado depresivo dura de forma ininterrumpida semanas o meses, el cuadro de gravedad depresiva es vivido por el afectado con sufrimiento e impide sus actividades personales y sociales, los estados de ánimo no se pueden cambiar mediante acontecimientos especiales en su entorno, o bien el afectado apenas reacciona a una dedicación especial de sus familiares (Freyberger, 1995).

Poco a poco se llega a los siguientes síntomas: pérdida de interés de las cosas, incapacidad para alegrarse o para participar en las actividades de otras personas, falta de decisión, intranquilidad o paralización internas, cavilación y estrechez de mente, abatimiento o cansancio, inhibición psicomotriz o pérdida de la energía, temores, falta de apetito, pérdida de peso, trastornos del sueño, diferentes dolencias físicas (sensación de presión en la cabeza y el vientre, sequedad de boca y estreñimiento).

El sentimiento de autoestima de las personas depresivas está condicionado por la enfermedad y es, generalmente, muy debilitado. La persona depresiva se puede sentir inservible y $\sin$ valor alguno, y a veces como si fuera una gran carga para su familia. Se acusa a sí misma de acciones que se sitúan muy lejos en el tiempo, que ahora se ven desde una perspectiva diferente y que a los familiares les parecen, por lo general, exageradas, irreales. Se recriminan a sí mismas faltas y delitos anteriores. El depresivo se siente malo y perdedor; en ocasiones ve el conjunto de su vida como a través de un velo depresivo (Freyberger, 1995).
Los hallazgos señalados revelan que la violencia de pareja es un factor que no solo se ha dado en familias que han permanecido viviendo en un solo lugar a lo largo de sus vidas, sino que también en mujeres que, junto con sus parejas e hijos, migran en búsqueda de nuevas oportunidades con la expectativa de mejorar sus estilos de vida. Pareciera ser que el desplazamiento geográfico asegura una mejora intrínseca, sin embargo, en muchos casos esto no resulta así, la dificultad que guarda el establecerse en una ciudad distinta enrarece la adecuada interacción entre los miembros de la pareja, potenciando la aparición de interacciones violentas y depresión, son fenómenos de alcance mundial según Larrain (1994). En este sentido, buscando alentar el debate sobre la afirmación de que la inmigración representa un cambio benéfico, cuando en realidad se trata de un cambio que encierra importantes factores de riesgo psicosocial a nivel personal y familiar, se plantearon los siguientes cuestionamientos: ¿existe relación entre la violencia de pareja y la depresión en mujeres madres inmigrantes que residen actualmente en el Estado de México? ¿Existe relación entre la depresión y la autoeficacia materna en mujeres inmigrantes que residen actualmente en el Estado de México? ¿Existirá relación entre la violencia de pareja y la autoeficacia materna en mujeres inmigrantes que residen actualmente en el Estado de México?

De esta manera, se planteó en este estudio como objetivo general detectar si existe relación entre la violencia de pareja y la depresión en mujeres inmigrantes que viven en el Estado de México. Como objetivos específicos: detectar si existe relación entre la violencia de pareja y la autoeficacia materna y detectar si existe relación entre la depresión y la autoeficacia materna en las mujeres inmigrantes que viven en este Estado. Derivándose tres hipótesis de trabajo: 1) La depresión es un elemento psicológico presente en mujeres inmigrantes víctimas de violencia de pareja que viven en el Estado de México; 2) La violencia de pareja es un factor que se 
relaciona con la ineficacia materna en mujeres inmigrantes que viven en la región mencionada; 3. La depresión es un elemento psicológico que se asocia con la ineficacia materna en mujeres inmigrantes que habitan en este Estado.

\section{Metodología}

El tipo de estudio aplicado fue correlacional explicativo, con el cual se buscó medir el grado de asociación entre las variables planteadas (Hernández, Fernández y Baptista, 2006).

\section{Variables}

Se trabajaron variables atributivas (Hernández et al., 2006; Rojas, 1993):

Violencia de pareja: todo acto dañino intencional (voluntario) emitido por un miembro de la pareja, acto que va dirigido en contra del otro miembro de la pareja que tiende hacia la destrucción de ese miembro o hacia el abuso de este; estos actos pueden ser físicos, económicos, sexuales o psicológicos; se manifiestan de manera directa o inversa teniendo repercusiones en la salud del receptor (Delgadillo y Mercado 2010).

Depresión: enfermedad que afecta el organismo (cerebro), el ánimo y la manera de pensar. Perjudica la forma en que una persona come y duerme; también cómo uno se valora a sí mismo (autoestima) y la forma en que uno piensa. Un trastorno depresivo no es lo mismo que un estado pasajero de tristeza. No indica debilidad personal. No es una condición de la cual uno puede liberarse voluntariamente. Presenta sentimientos persistentes de tristeza, ansiedad o vacío; de desesperanza y/o pesimismo; de culpa, inutilidad, y/o impotencia; puede haber irritabilidad, inquietud; pérdida de interés en las actividades o pasatiempos que antes disfrutaba, incluso, en adultos, las relaciones sexuales; fatiga y falta de energía; dificultad para concentrarse, recordar detalles y para tomar decisiones; se pueden producir pensamientos suicidas o intentos de suicidio; dolores y malestares persistentes, dolores de cabeza, cólicos o problemas digestivos que no se alivian incluso con tratamiento (Instituto Nacional de la Salud Mental, NIMH, 2011).

Autoeficacia materna: medida de autoeficacia del desempeño sobre el control percibido sobre la salud de los niños, habilidades sociales y el desarrollo cognitivo como responsable del bienestar y crecimiento del menor de quien se es responsable (Diener, Nievar \& Wright, 2003).

\section{Muestra}

No probabilística, las participantes fueron contactadas a través de las instituciones de atención a mujeres víctimas de violencia familiar (Hernández et al., 2006; Kerlinger, 1988). En total se trabajó con 40 mujeres inmigrantes que viven en el Estado de México. Criterios de selección: 1) Mujeres inmigrantes que hayan vivido al menos un episodio de violencia doméstica; 2) Mujeres inmigrantes que tengan un hijo en preescolar; 3) Mujeres inmigrantes con edades entre los 20 y los 60 años; 4) Mujeres que hayan inmigrado a Toluca al menos desde hace dos años y no más de 25 .

\section{Instrumentos}

The Revised Conflict Tactic Scale: la versión revisada del conflicto Táctica Escala (CTS2) (Straus, Hamby, Boney-McCoy \& Sugarman, 1996) mide la violencia de pareja. Esta medida autoinforme enumera los actos de malos tratos psicológicos y físicos recibidos por su par durante el año pasado. Los coeficientes de fiabilidad interna son de 0,79 para la agresión psicológica y 0,86 por asalto físico (Straus et al., 1996). Estas escalas se han utilizado con poblaciones que hablan español con una fiabilidad interna satisfactoria (Connelly, Newton, \& Aarons, 2005). 
The Center for Epidemiological SurveyDepression Scale: Escala de Depresión (Radloff, 1977) es una medida de autoinforme de 20 ítems y ha sido ampliamente utilizado en estudios de investigación y como instrumento de cribado para la depresión clínica. Estimaciones de la fiabilidad interna oscilan entre 0,85 y 0,90 y son similares a los europeo-americanos, afroamericanos y mexicano-americanos (Caetano \& Cunradi, 2003).

The Parental Involvement and Efficacy: es una medida de autoeficacia materna que se ocupa del control percibido sobre la salud de los niños, las habilidades sociales y el desarrollo cognitivo (Diener, et al., 2003). También evalúa las percepciones de las madres de su posible eficacia como padres. Estos puntos se califican en una escala de Likert que va desde totalmente en desacuerdo a totalmente de acuerdo. Una puntuación alta indica una creencia fuerte en la autoeficacia materna y participación. Esta medida fue normada en un estudio multiestado, con alphas que van 0,81 a 0,84 (Nievar, BrophyHerb, Fitzgerald \& Diener, 2007). La versión en español se incluyó en el estudio de validación.

\section{Diseño de investigación}

El diseño de estudio utilizado fue no experimental transeccional (Hernández, et al., 2006).

\section{Procedimiento}

La aplicación de los cuestionarios se realizó en forma individual y privada en las instalaciones de las instituciones que brindan la atención a las mujeres víctimas de violencia, garantizando la confidencialidad y anonimato de los datos. Se les hizo saber en un primer momento en qué consistía el estudio para que otorgaran su consentimiento informado; una vez obtenido, se les daba las instrucciones sobre el llenado de los instrumen- tos. El tiempo promedio de aplicación fue de 40 minutos.

\section{Captura de información}

Los pasos para la captura de la información fueron los siguientes: 1) Aplicación de los instrumentos; 2) Elaboración de la base de datos; 3) Análisis estadísticos de los datos; 4) Interpretación de los resultados; 5) Presentación de resultados; 6) Realización de conclusiones y sugerencias de la investigación.

\section{Procesamiento de la información}

Los datos se corrieron mediante el Coeficiente de Correlación de Pearson, con $\mathrm{P} \leq 0,05$

\section{Resultados}

Las características generales de las participantes fueron, en edad, un promedio de 33,2 años, con una mediana de 32 años en un rango de 21 a 49 años. En el estado civil actual predominaron las mujeres casadas, seguidas de las mujeres separadas y por último las mujeres en unión libre. Ver tabla 1, Estado civil.

\section{Tabla 1 \\ Estado civil}

\begin{tabular}{|lr|}
\hline \multicolumn{1}{|c}{ Estado civil } & Porcentaje \\
\hline Casadas & $82,22 \%$ \\
\hline Separadas o divorciadas & $11,11 \%$ \\
\hline Unión libre & $06,66 \%$ \\
\hline
\end{tabular}

Fuente: Vargas y Delgadillo

En cuanto a la escolaridad, hubo una mayor frecuencia en las mujeres con estudios de preparatoria, seguida de estudios de secundaria, licenciatura, carrera técnica, primaria y, por último, de maestría o posgrado. Ver tabla 2, Escolaridad. 
Tabla 2

Escolaridad

\begin{tabular}{ll}
\multicolumn{1}{c}{ Escolaridad } & Porcentaje \\
\hline Primaria & $15,55 \%$ \\
\hline Secundaria & $20,00 \%$ \\
\hline Preparatoria & $24,44 \%$ \\
\hline Carrera técnica & $17,70 \%$ \\
\hline Licenciatura & $20,00 \%$ \\
\hline Maestría o posgrado & $02,22 \%$ \\
\hline
\end{tabular}

Fuente: Vargas y Delgadillo

Sobre la ocupación, el mayor número de mujeres se ubicó en la categoría de ama de casa, seguida de tener un empleo, negocio propio y por último estudiante. Ver tabla 3, Ocupación.

Tabla 3

Ocupación

\begin{tabular}{ll} 
Ocupación & Porcentaje \\
\hline Hogar & $44,44 \%$ \\
\hline Estudiante & $02,22 \%$ \\
\hline Negocio propio & $11,11 \%$ \\
\hline Empleada & $42,22 \%$ \\
\hline
\end{tabular}

Fuente: Vargas y Delgadillo

Referente al número de hijos, la mayor frecuencia de mujeres se ubicó en la categoría de dos hijos, seguida de tres, uno, cuatro y cinco hijos respectivamente. Ver tabla 4, Número de hijos.
Tabla 4

Número de hijos

\begin{tabular}{|lc|}
\hline \multicolumn{1}{|c}{ Número de hijos } & Porcentaje \\
\hline Un hijo & $08,88 \%$ \\
\hline Dos hijos & $51,11 \%$ \\
\hline Tres hijos & $28,88 \%$ \\
\hline Cuatro hijos & $08,88 \%$ \\
\hline Cinco hijos & $02,22 \%$ \\
\hline
\end{tabular}

Fuente: Vargas y Delgadillo

En total, las entrevistadas reportaron 11 distintos lugares de procedencia, predominando los municipios del Estado de México, seguido del Estado de Michoacán, el Distrito Federal, Jalisco, Hidalgo, Veracruz, Morelos, Guerrero, Puebla, Chihuahua y Nayarit. Ver tabla 5, Lugar de procedencia.

Tabla 5

Lugar de procedencia

\section{Lugar de procedencia $\quad$ Porcentaje}

Distrito Federal $\quad 06,66 \%$

Puebla $\quad 02,22 \%$

\begin{tabular}{|l|l|}
\hline Michoacán & $11,11 \%$ \\
\hline Municipios del Estado de México & $51,11 \%$ \\
\hline Chihuahua & $02,22 \%$ \\
\hline Jalisco & $06,66 \%$ \\
\hline Hidalgo & $04,44 \%$ \\
\hline Nayarit & $02,22 \%$ \\
\hline Veracruz & $04,44 \%$ \\
\hline Morelos & $04,44 \%$ \\
\hline Guerrero & $04,44 \%$ \\
\hline
\end{tabular}

Fuente: Vargas y Delgadillo 
El tiempo de residencia actual presentó un promedio de 11,33 años, con una mediana de 10 años, en un rango de cinco a 25 años, mientras que el tiempo de residencia en su hogar anterior presentó un promedio de 20,48 años, con una mediana de 20 años en un rango de 11 a 30 años.
A partir de la aplicación y resultados de los instrumentos: The Parental Involvement and Efficacy, The Center for Epidemiological Survey-Depression Scale y The Revised Conflict Tactic Scale, que midieron la autoeficacia materna, la depresión y la violencia de pareja respectivamente, se obtuvieron los siguientes resultados.

Tabla 6

Relación entre la violencia general de pareja, depresión y autoeficacia materna

\begin{tabular}{|lcccc|}
\hline & Correlaciones & Desempeño parental & Depresión & Violencia \\
\hline \multirow{2}{*}{$\begin{array}{l}\text { Desempeño } \\
\text { parental }\end{array}$} & $\begin{array}{c}\text { Pearson Correlation } \\
\text { Sig. (2-tailed) }\end{array}$ & 1000 & .558 & .302 \\
& $\mathrm{~N}$ & & .000 & .044 \\
\multirow{2}{*}{ Depresión } & Pearson Correlation & .558 & 45 & 45 \\
& Sig. (2-tailed) & .000 & 1000 & .514 \\
& $\mathrm{~N}$ & 45 & & .000 \\
\hline \multirow{3}{*}{ Violencia } & Pearson Correlation & .302 & .514 & 45 \\
& Sig. (2-tailed) & .044 & .000 & 1000 \\
\hline
\end{tabular}

Fuente: Vargas y Delgadillo

Se puede observar en la tabla 6 , Relación entre la violencia general de pareja, depresión y autoeficacia materna, que la relación es baja entre la variable Violencia y Desempeño Parental de .302; la relación entre Violencia y Depresión Parental moderada .514; y la relación entre Depresión y Desempeño Parental moderada .558.
Para un mejor análisis de la variable violencia se desagregó la violencia de tipo psicológica y la violencia de tipo física. Ver tabla 7, Relación entre la violencia física y psicológica con la depresión y autoeficacia materna:

Tabla 7

Relación entre la violencia física y psicológica con la depresión y autoeficacia materna

\begin{tabular}{|c|c|c|c|c|c|}
\hline \multicolumn{2}{|c|}{ Correlaciones } & $\begin{array}{l}\text { Desempeño } \\
\text { parental }\end{array}$ & Depresión & $\begin{array}{l}\text { Violencia } \\
\text { Psicológica }\end{array}$ & $\begin{array}{l}\text { Violencia } \\
\text { física }\end{array}$ \\
\hline $\begin{array}{l}\text { Desempeño } \\
\text { parental }\end{array}$ & $\begin{array}{c}\text { Pearson Correlation } \\
\text { Sig. (2-tailed) } \\
\text { N }\end{array}$ & & & $\begin{array}{c}.208 \\
170 \\
45\end{array}$ & $\begin{array}{c}.339 \\
.023 \\
45\end{array}$ \\
\hline Depresión & $\begin{array}{c}\text { Pearson Correlation } \\
\text { Sig. (2-tailed) } \\
\text { N }\end{array}$ & & & $\begin{array}{c}.472 \\
.001 \\
45\end{array}$ & $\begin{array}{l}.462 \\
.001 \\
45\end{array}$ \\
\hline $\begin{array}{l}\text { Violencia } \\
\text { psicológica }\end{array}$ & $\begin{array}{l}\text { Pearson Correlation } \\
\text { Sig. (2-tailed) }\end{array}$ & $\begin{array}{l}.208 \\
.170 \\
45\end{array}$ & $\begin{array}{l}.472 \\
.001 \\
45\end{array}$ & 1000 & $\begin{array}{l}.650 \\
.000 \\
45\end{array}$ \\
\hline
\end{tabular}

Fuente: Vargas y Delgadillo 
Relación baja entre desempeño parental y violencia física de .339. Relación moderada entre depresión y violencia psicológica y depresión y violencia física de .472 y .462 respectivamente. Relación alta entre la variable de violencia psicológica y violencia física de .650 .

\section{Discusión}

Esta investigación se sitúa desde una interrogante, la existencia de relación entre la violencia de pareja, la depresión y la autoeficacia materna en las mujeres inmigrantes que viven en el Estado de México. Los resultados arrojaron en lo general correlaciones moderadas. Específicamente en lo que se refiere a la correlación moderada entre la variable violencia y la variable depresión, esto admite, mesuradamente, afirmar que la violencia produce efectos negativos en el estado anímico de estas mujeres. Las correlaciones obtenidas entre, por un lado, la violencia psicológica y la depresión, y por otro lado, la violencia física y la depresión permiten confirmar la liga en términos específicos de los efectos de la violencia de pareja sobre las mujeres como víctimas. De esta manera, no resulta difícil entender el por qué la alta correlación entre la violencia física y la psicológica, ambas manifestaciones componen un dispositivo dañino de control sobre la mujer víctima de la violencia de su pareja.

También se puede advertir cómo se encuentran asociadas las variables de desempeño parental y de depresión, se puede afirmar que un estado anímico desfavorable en las mujeres madres inmigrantes de hijos(as) pequeños(as) afecta el control percibido sobre la salud de los niños, habilidades sociales y el desarrollo cognitivo como responsable del bienestar y crecimiento del menor de quien se responsabiliza. Coincidiendo con el estudio de la Organización Panamericana de la Salud (2003), que indica que las víctimas de maltrato manifiestan más problemas emocionales que las que no son abusadas y una de estas manifestaciones es la depresión.
Este hallazgo resulta consistente con otros estudios en los que se afirma que las madres en relaciones violentas de pareja experimentan más estrés en el cuidado de sus hijos(as) (Holden \& Ritchie, 1991; Taylor, et al., 2009). Lo que no deberá disociarse del efecto que tiene sobre los niños(as), quienes al observar el uso de la violencia como una forma de resolver conflictos experimentan resultados negativos (Campbell, et al., 1995).

En cuanto a la asociación entre la violencia y el desempeño parental no se puede afirmar que se produzca una fuerte liga entre estas variables, lo que acaso pudiese explicarse con base en las habilidades para separar los dos subsistemas. El subsistema de pareja y el subsistema madre-hijo(a). Aunque habría que estar alertas como investigadores al hecho de que las mujeres participaron en este estudio sin estar trabajando con el investigador algún tipo de encuadre clínico de intervención como víctimas de violencia de pareja, y por otro lado, el número de participantes no se correspondió plenamente a una gran muestra. En el mismo sentido, se puede explicar la baja relación entre la violencia física y el desempeño parental.

Sin embargo, habrá que tener presente lo que apunta la Encuesta Nacional sobre la Violencia contra las Mujeres, Envim (Instituto Nacional de Salud Pública, 2003), la mujer, como responsable de muchas más obligaciones que el hombre, frecuentemente se siente en falta y abrumada por sentimientos de culpa. Lo que conduce a justificar el maltrato de la pareja y favorecer el que ella lo soporte, porque el maltrato resulta "merecido". Con frecuencia se relata que la pareja, después de maltratarla, exige que sea ella quien pida perdón. Esto cumple la función de establecer la certeza sobre quién es el que manda y quien la que obedece". Y efectivamente, en la información que proporcionaron las víctimas mediante los instrumentos de depresión, muchas veces se sienten culpables por la actitud agresiva del cónyuge, ya que el incumplimiento de sus deberes como esposas o 
el hecho de no obedecer a su marido provocan que se enfurezca y que las golpeen.

Con base en la literatura revisada, es importante hacer mención, que éste sentido de culpa interiorizado por la víctima no solo es causado por el agresor, sino también por los cimientos culturales fuertemente arraigados por la sociedad patriarcal, causante de que la mujer ocupe un segundo lugar, ocupándose de las tareas domésticas, trabajo invisible que carece de reconocimiento social, mientras que el hombre debe ser capaz de sobrellevar sus problemas sin ayuda de nadie. Gilmore (1995) menciona al respecto que todas las sociedades distinguen entre lo masculino y lo femenino, proporcionan papeles sexuales aprobados para los hombres y mujeres en edad adulta, entre estas diferencias está la superioridad física del hombre hacia la mujer, dando pauta a la tolerancia de la violencia del hombre hacia ella. Lo que pudiera variar a causa de los matices de acuerdo a la cultura de origen y a la cultura receptora para el caso de las mujeres migrantes.

La mayoría de las entrevistadas dijeron encontrar más diferencias en la forma de pensar de la gente que viene de pequeños pueblos a la gente que viene de pueblos más grandes, de ciudades pequeñas y de grandes ciudades, lo que muestra cierta correspondencia entre algunos elementos teóricos que afirman que en el marco de la violencia el poder debe ser conceptualizado como la capacidad de definir aquello que será considerado normal y válido, generando prácticas que se mantienen y a la vez refuerzan patrones sociales, interacciónales, conductuales y lingüísticos creados y mantenidos por la cultura, sin permitir alternativas y negando los derechos del otro, generando injusticias y desigualdades (Sarquis, 1995).

\section{Conclusiones}

De acuerdo con la información encontrada, es posible determinar que una persona que vive violencia de pareja también sufre en alguna medida de depresión, este trabajo ha revelado que:

La violencia doméstica hacia la mujer sucede y prevalece tanto en las familias que han permanecido viviendo en un solo lugar como en las familias que han migrado. Mujeres de distintas edades y ocupaciones con o sin hijas/os, casadas o no, los agresores van desde los esposos, parejas, pasando por exesposos y exparejas.

En todos los casos la violencia física va acompañada de la violencia psicológica en forma de intimidación y abuso verbal, sin embargo, la violencia psicológica no siempre va acompañada de violencia física.

Existe cierta asociación entre las variables medidas (autoeficacia materna, depresión y violencia) las cuales son: a mayor violencia, mayor depresión, o bien, a menor violencia menor depresión; aunque con una correlación baja, es posible esperar una menor autoeficacia materna conforme se produzca una mayor violencia de pareja; a mayor depresión, menor autoeficacia materna, o bien, a menor depresión, mayor autoeficacia materna. Por lo tanto la violencia origina depresión en las mujeres migrantes, esta a su vez, al igual que la violencia, produce una baja en la autoeficacia materna en mujeres migrantes violentadas.

Desglosando la violencia en violencia física y violencia psicológica, se concluye que la violencia física influye hasta cierto punto en la presentación de síntomas depresivos, sin influir directamente en la autoeficacia materna. Igualmente, la violencia psicológica puede originar síntomas depresivos, sin influir directamente en la autoeficacia materna. Como tampoco la violencia psicológica se acompaña forzosamente de la violencia física.

Todo lo anterior mantiene vigente la afirmación aquella que indica que la violencia contra la mujer no debería ser un asunto ínti- 
mo ni privado, no debería ser un problema de relaciones interpersonales limitado a la pareja, si no que debiera tratarse como un problema público, social y cultural. Siendo urgente el establecimiento de programas sociales preventivos y correctivos para esta población, mujeres migrantes. Si bien el diseño de este estudio es transeccional y no se puede hablar de causalidad en términos metodológicos, sus resultados pueden ser útiles para el diseño y rediseño de mejores programas.

\section{Referencias}

Álvarez, M. (2003). Violencia de género y adolescencia, una propuesta de interpretación educativa. Otras miradas, diciembre, 3(002), 96-110. Recuperado de http:// www.redalyc.com/

Beck, A. T., Rush, A. J., Shaw, B. F., y Emery, G. (1983). Terapia cognitiva de la depresión. Bilbao: Desclée de Brouwer.

Boneva, B. S., \& Frieze, I. H. (2001). Toward a concept of a migrant personality. Journal of Social Issues, 57(3), 477-491.

Caetano, R., \& Cunradi, C. B. (2003). Intimate partner violence and depression among Whites, Blacks and Hispanics. Annals of Epidemiology, 13, 661-665.

Campbell, J. C., Sullivan, C. M., \& Davidson, W. S. (1995). Women who use domestic violence shelters: Changes in depression over time. Psychology of Women Quarterly, 19, 237-255.

Connelly, C. D., Newton, R. R., \& Aarons, G. A. (2005). A psychometric examination of English and Spanish versions of the Revised Conflict Tactics Scale. Journal of Interpersonal Violence, 20, 1560-1579.

Delgadillo, L., y Mercado, A. (2010). Violencia laboral, una realidad incomoda. México:
Universidad Autónoma del Estado de México.

Delgadillo, L. (2011). Mujeres cautivas entre el amor y el odio. Una exploración psicosocial de la violencia de pareja. España: Editorial Académica Española.

Diener, M. L., Nievar, M. A., \& Wright, C. (2003). Attachment security among mothers and their young children living in poverty: Associations with maternal, child, and contextual characteristics. Merrill-Palmer Quarterly: Journal of Developmental Psychology, 49(2), 154182.

Ferreira, G. (1989). La mujer maltratada. Buenos Aires: Sudamericana.

Freyberger, H. (1995). La depresión. Bilbao: Mensajero.

Gilmore, D. (1995). Hacerse hombres. Argentina: Ed. Paidós.

Heise, L., y García-Moreno, C. (2003). 'La violencia en la pareja', en Organización Panamericana de la Salud, Informe mundial sobre la violencia y la salud. Publicación científica y técnica, 588, 97-131. Washington.

Hernández, R., Fernández, C., y Baptista, P. (2006). Metodología de la investigación. México: McGraw Hill.

Holden, G. W., \& Ritchie, K. I. (1991). Linking extreme marital discord, child rearing and child behavior problems: evidence from battered women. Child Development, 62, 311-327.

Instituto Nacional de Estadística, Geografía e Informática. (2004). Encuesta nacional sobre la dinámica de las relaciones en los hogares 2003. México: INEGI. 
Instituto Nacional de Salud Pública. (2003). Encuesta nacional sobre violencia contra las mujeres. México.

Jacobson, N., y Gottman, J. (2001). Hombres que agreden a sus mujeres. Cómo poner fin a las relaciones abusivas. México: Ed. Paidós.

Kerlinger, F. (1988). Investigación del comportamiento. México: Mc Graw Hill.

Larraín, S. (1994). Violencia, puertas adentro: la mujer golpeada. Santiago: Universitaria.

Minuchin, S. (2004). Familias y terapia familiar. México: Gedisa.

National Institute of Mental Health. (2011). Recuperado de http://www.nimh.nih.gov/ science-news/2011/index.shtmlhttp:// www.medigraphic.com/espanol/e-htms/ e-imss/e-im2007/e-im07-3/em-im073d. html.

Nievar, M. A., Brophy-Herb, H., Fitzgerald, H., \& Diener, M. (2007). Parenting in early childhood: Validation of parental involvement and efficacy. Poster session presented at the American Educational Research Association Annual Meeting. Chicago, IL.

Patró, R., Limiñana, R. M., y Martínez, F. (2003). Valores asociados a la violencia en hijos de mujeres maltratadas. Póster presentando en el IV Congreso Mundial de Educación Infantil y Formación de Educadores. Málaga (España).

Rojas, S. (1993). Formación para investigadores educativos. México: Plaza y Valdés.

Sarquis, C. (1995). Formulación de politicas para incrementar la participación de la mujer en la administración pública. México: M. A. Porrúa.

Satir, V. (1994). Psicoterapia familiar. México: Prensa Medica Mexicana.

Straus, M. A., Hamby, S. L., Boney-McCoy, S., \& Sugarman, D. B. (1996). The Revised Conflict Tactics Scales: Development and preliminary psychometric data. (STS2). Journal of Family Issues, 17 (3), 283-316.

Radloff, L. S. (1977). The CES-D CES-D scale: A self-report depression scale for research in the general population. Applied Psychological Measurement, 1, 385-401.

Taylor, C. A., Guterman, N. B., Lee, S. J., \& Rathouz, P. J. (2009). Intimate Partner Violence, Maternal Stress, Nativity and Risk for Maternal Maltreatment of Young Children. American Journal of Public Health, 99(1), 175-183.

Widlöcher, D. (1986). Las lógicas de la depresión. Barcelona: Herder. 
